\title{
Landmark green light for Mosquirix malaria vaccine
}

In a major public health milestone, European regulators have for the first time delivered a positive scientific opinion of a vaccine against malaria. The European Medicines Agency (EMA)'s decision paves the way for a policy recommendation by the World Health Organization (WHO) for vaccinating children in sub-Saharan Africa. The vaccine, Mosquirix (also known as RTS,S/AS01), from Londonbased GlaxoSmithKline, is the result of three decades of development. Although the vaccine's efficacy is limited, its public health value could be particularly great for children in hightransmission areas where mortality is very high.

GSK received the regulatory nod from the EMA's Committee for Medicinal Products for Human Use in late July. "It's a big scientific achievement," says David Kaslow, vice president of product development at PATH, an international nonprofit organization for global health, located in Seattle, Washington. "This is the first time a vaccine has shown a favorable benefitrisk profile against malaria," says Kaslow.

The European agency recommended Mosquirix to prevent malaria caused by the parasite Plasmodium falciparum in children aged 6 weeks to 17 months. The positive opinion provides much-needed proof that a vaccine is possible. GSK ran multicenter trials in partnership with the PATH Malaria Vaccine Initiative in 15,459 participants at 11 sites in seven subSaharan African countries: Burkina Faso, Gabon, Ghana, Kenya, Malawi, Mozambique and Tanzania.

"It was never entirely clear if it was biologically possible to develop a malaria vaccine," says Kaslow. The parasite has 5,000 genes and three distinct life cycle stages, which makes it difficult to identify targets for the immune system to attack. "This is a wily parasite," he says, one that has evolved and shifted in response to malarial therapies and insecticides. Work in the 1980s by wife-and-husband team of Ruth and Victor Nussenzweig at New York University first showed that the target for protective antibodies is the circumsporozoite protein (CSP) on the of sporozoites at the liver stage, after the malaria parasite is inoculated into humans. This discovery set the stage for a human vaccine.

But more than a dozen different vaccines made using different technologies, including synthetic peptides, DNA constructs, adjuvants and delivery systems, failed to offer enough protection. Although all induced low levels of antibodies it became clear that to increase protection, the vaccine had to generate a more potent antibody response and a T-cell response.

Mosquirix is the first vaccine to demonstrate an immune response resulting in increased efficacy in humans. It is based on antigens that makeup the RTS acronym-the central tandem repeat (R) from the CSP, epitopes from the CSP carboxy-terminal (T); and the surface (S) antigen of hepatitis B virus-genetically engineered into the hepatitis B surface antigen (HBsAg) platform. When co-expressed in yeast, the fusion protein spontaneously forms virus-like particles with free HBsAgs, which are taken up by antigen-presenting cells, priming the immune system.

The vaccine's novel adjuvant also stimulates $\mathrm{T}$ helper cell responses and further activates macrophages and other antigen-presenting cells. The adjuvant AS01 is a liposomal formulation of the monophosphoryl lipid A and Quillaja saponaria fraction 21, the latter an extract from the soap bark tree, a Chilean evergreen. Together, vaccine and adjuvant prompt an immune response designed to intercept the parasite in circulation before it travels to the liver, where it multiplies, eventually releasing parasites back into the bloodstream and causing malarial symptoms.

Overall, it's likely antibodies to CSP carry most, if not all, of the parasite-fighting load, though a small response by $\mathrm{CD} 8^{+} \mathrm{T}$ cells can't be ruled out, says Eleanor Riley, professor of infectious disease immunology at the London School of Hygiene and Tropical Medicine. Protection is mostly down to higher antibody titers to CSP, as these correlate to better vaccine responses. That response with Mosquirix is "about as good as it's going to get," she says. "To make this vaccine better it would be necessary to add in more antigens to broaden the immune response and to really target in the liver those few sporozoites that make it there."

GSK began work on the present RTS,S vaccine in the late 1980s, in conjunction with the Walter Reed Medical Research Institute in Silver Spring, Maryland. The British drug-maker spent much of the 1990s working to improve the performance of the vaccine by tweaking the adjuvant, eventually incorporating the AS01 adjuvant system in the later part of that decade. Since 2001, the vaccine has been developed by GSK and PATH, with \$200 million in funding from the Bill and Melinda Gates Foundation. GSK says it has invested $\$ 350$ million so far developing Mosquirix and expects to spend an additional \$260 million to complete the project.

GSK published final phase 3 trial results for Mosquirix in April 2015 (Lancet 386, 31-45, 2015). A three-dose schedule of the vaccine followed by a booster 18 months later reduced the number of cases of clinical malaria by $36 \%$ in children aged $5-17$ months and by $26 \%$ in infants aged 6-12 weeks, over an average 48 months and 38 months across trial sites,

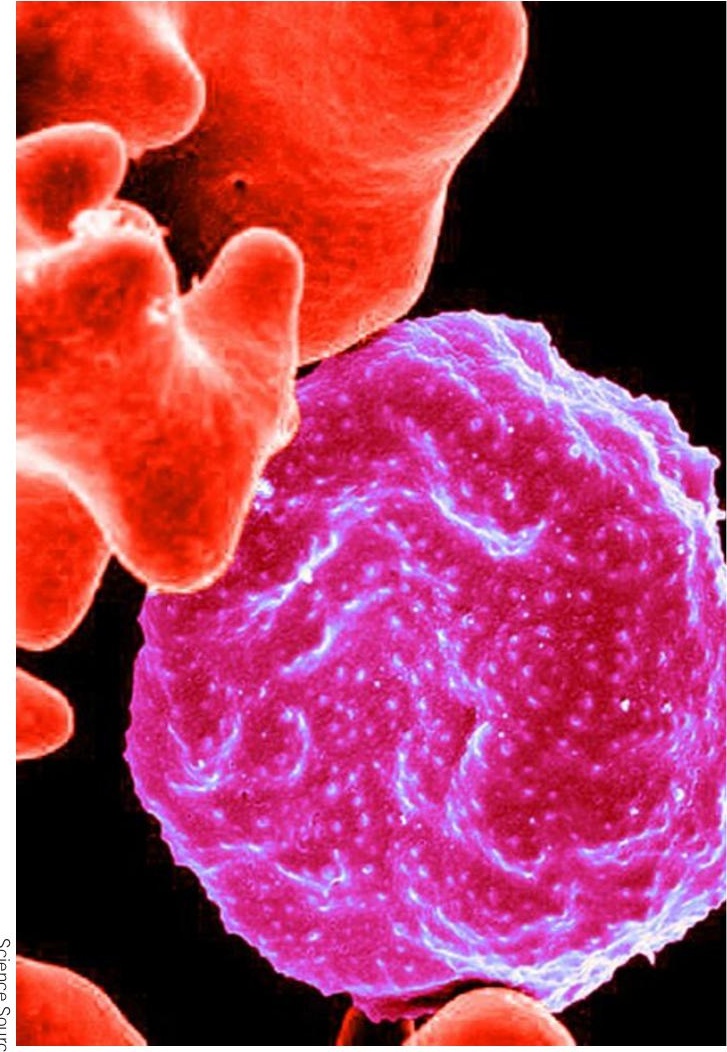

A red blood cell infected with malaria parasites.

respectively. The booster dose appears to be critical for the vaccine's success; without it, the vaccine failed to demonstrate statistical superiority over existing malaria control efforts, including insecticide-treated bed nets. Because the booster clearly affects vaccine efficacy, there's some chance that "regimen optimization," tweaking the timing or number of the Mosquirix injections, could further improve its performance, notes Annie Mo, program officer for parasite vaccine development at the US National Institutes of Health's National Institute for Allergy and Infectious Diseases in Bethesda, Maryland.

Moncef Slaoui, the chairman of global vaccines at GSK and a scientist who has been involved with Mosquirix development for 30 years, describes the vaccine's efficacy as "statistically significant but modest." But because "malaria is incredibly endemic in these regions, with an extraordinary attack rate," even a modestly effective vaccine can have an important impact, he says. In the GSK trial, Mosquirix prevented 1,700 cases of malaria for every 1,000 toddlers in the trial over the four-year time frame, he says (at some trial centers, that figure was as high as 6,000 episodes prevented per 1,000 children). In infants, vaccination 
prevented about one case of malaria per child over the same period.

According to the WHO's 2014 world malaria report (http://www.who.int/malaria/publications/world_malaria_report_2014/en/), there were 198 million cases of malaria worldwide in 2013 and the disease killed an estimated 584,000 people. Of those deaths, $90 \%$ occurred in sub-Saharan Africa, where $83 \%$ were children under the age of 5. Extrapolating the vaccine's success to the entire region, even at the onecase-per-child rate, "that's 30 million cases of malaria prevented" every year for three or four years, Slaoui says. Given the significant burden of treating those children, the vaccine could be "an enormous benefit to public health."

As for why the vaccine works some of the time but far from all of the time, Slaoui notes the tenacity and ubiquity of the parasite. "On average, a child [in sub-Saharan Africa] gets a malaria-infected mosquito bite about once a week," he says. "You can protect against 51 of those bites over a year and fail to protect for one and the child might get malaria. It's an incredibly high hurdle."

The European regulator reviewed the vaccine under Article 58, which allows the agency to recommend a vaccine or medicine for use outside the European Union. With this endorsement in place, the WHO can now formulate a policy recommendation, and individual nations can determine how to use the vaccine-and how to fund its use-alongside existing malaria efforts. The WHO's Strategic Advisory Group of Experts on Immunization and its Malaria Policy Advisory Committee are scheduled to meet in November 2015.

A future malaria vaccine arsenal might include a so-called heterologous prime-boost approach, whereby two different vaccines containing the same antigen are given sequentially to drive a greater immune response to the parasite. Several prime-boost trials are ongoing, though a combination of RTS,S and an adenoviral vector expressing CSP from Johnson \& Johnson's Crucell vaccine division in Leiden, The Netherlands, was no more effective than Mosquirix alone, according to recently published results of a phase 2 trial (PLoS One 10, e0131571, 2015). A prime-boost strategy from a group led by Adrian Hill, director of the Jenner Institute in Oxford, UK, has shown promise in early trials using a different sporozoite antigen, ME-TRAP (multiple epitope-thrombospondin-related adhesion protein) (J. Infect. Dis. 211, 1076-1086, 2015.).

WHO spokesperson Gregory Härtl calls the vaccine a "big step forward," noting that its success will be held up as a yardstick for subsequent attempts at preventing the disease. But there's room for a single malaria vaccine with greater efficacy. "We have to find the right targets, the right antigens for $\mathrm{T}$ cells to recognize and use as a homing beacon for clearing the pathogen," says Chip Clark, CEO of Cambridge, MA-based Genocea Biosciences. Genocea's discovery efforts in malaria are supported by the Bill and Melinda Gates Foundation. The company's platform allows it to profile individuals' T-cell responses to the parasite and identify differences. With this strategy, the company hopes to find key T-cell antigens that could be added to an existing vaccine like Mosquirix or form the basis of something new. Among other approaches in early testing highlighted by PATH's Kaslow are a live, attenuated vaccine developed by Rockville, Maryland-based Sanaria that may generate a strong response to the parasite in the liver. Another strategy is a vaccine to block transmission of the parasite within the mosquito midgut.

GSK is developing a second-generation version of Mosquirix, which so far in early-stage trials in healthy volunteers is demonstrating "much higher" efficacy than what the company observed with Mosquirix at the same stage of development, says GSK's Slaoui. "It's a tweak," he says. "A highly scientific tweak with a strong rationale behind it. It's remarkable."

Chris Morrison Yardley, Pennsylvania

\section{Around the world in a month}

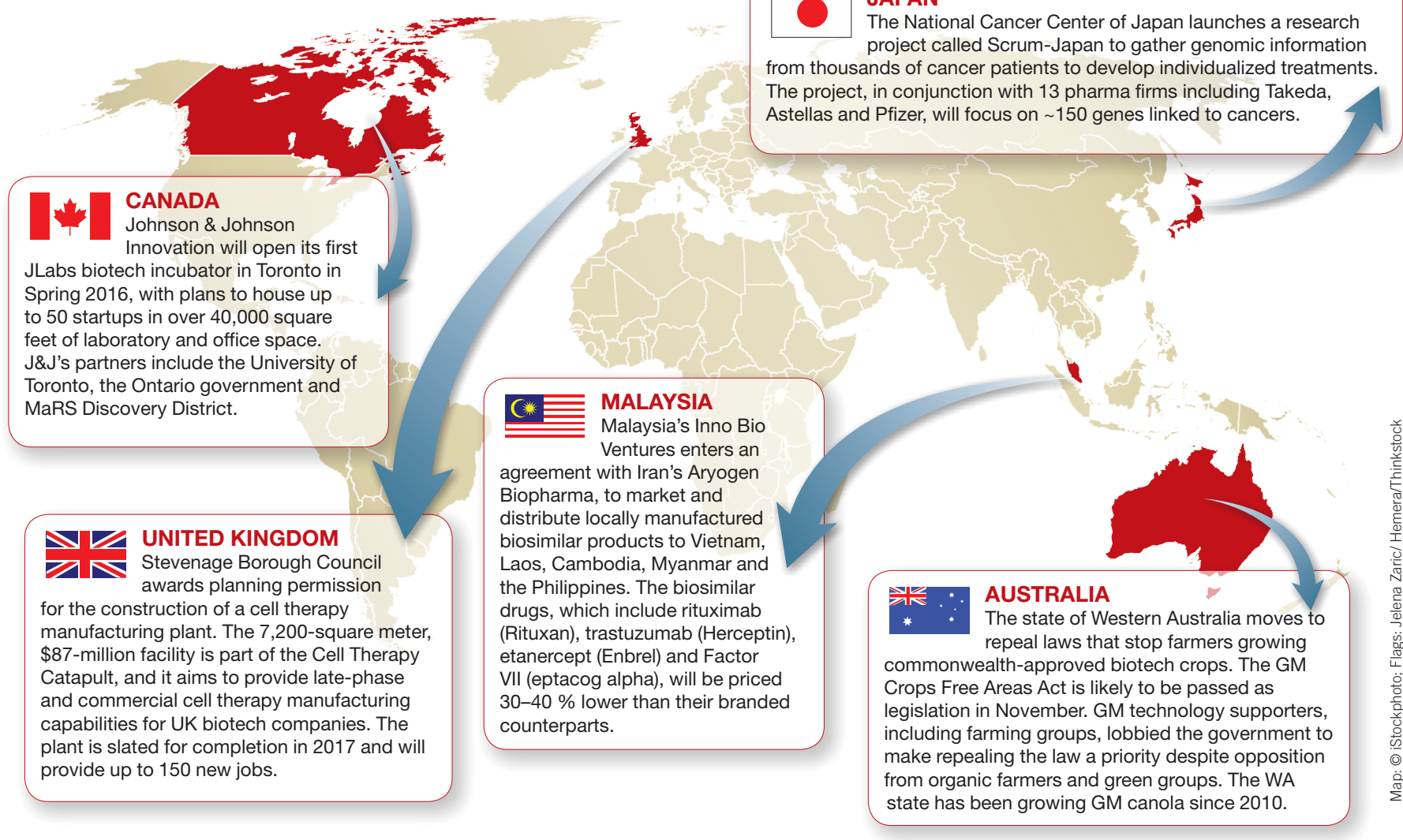

